TITLE:

\title{
Comparison of the clinical features of suicide attempters by jumping from a height and those by self- stabbing in Japan.
}

\section{$\operatorname{AUTHOR}(S):$}

Kimura, Ryo; Ikeda, Shunichiro; Kumazaki, Hirokazu; Yanagida, Makoto; Matsunaga, Hidenori

\section{CITATION:}

Kimura, Ryo ...[et al]. Comparison of the clinical features of suicide attempters by jumping from a height and those by self-stabbing in Japan.. Journal of affective disorders 2013, $150(2): 695-698$

ISSUE DATE:

2013-09-05

URL:

http://hdl.handle.net/2433/178755

\section{RIGHT:}

(c) 2013 Elsevier B.V.; この論文は出版社版でありません。引用の際には 出版社版をご確認ご利用ください。; This is not the published version. Please cite only the published version. 


\begin{abstract}
Background: A history of psychiatric disorders is a high risk for suicide. The present study compared the clinical features of psychiatric patients in Japan who attempted suicide by jumping from a height and those who attempted suicide by self-stabbing.

Methods: We compared two groups of suicide attempters who were hospitalized for both physical and psychiatric treatment $(n=202)$. We compared the psychiatric diagnoses and clinical features between those who attempted suicide by jumping from a height $(\mathrm{N}=147)$ and those who did so by self-stabbing $(\mathrm{N}=55)$.
\end{abstract}

Results: The self-stabbing group (mean age 52.3 years) was significantly older compared to the jumping group (mean age 37.9 years). A significantly higher proportion of females were found in the jumping group. Jumping from a height was significantly associated with schizophrenia spectrum disorders, whereas self-stabbing was significantly associated with mood disorders.

Limitations: The results were drawn from data from a single hospital in a large urban city, and the study population did not include subjects who completed their suicide attempts.

Conclusions: Our findings show that differences in suicide methods (here, between jumping from a height and self-stabbing) may be related to suicide attempters' psychiatric diagnosis, gender and age. It is thus important to obtain a more detailed background information about a patient's suicide attempt and to create suicide prevention plans in accord with individuals' psychiatric diagnosis, age and gender, especially among those who have attempted suicide by jumping from a height or self-stabbing.

\title{
Keywords:
}

Suicide attempts, jumping, self-stabbing

\section{Introduction}


Suicide is a major public health problem across the world. In Japan, the number of people who attempt suicide each year has remained over 30,000 (approx. 24 complete suicides per 100,000 persons) since 1998. Several factors have been suggested as contributors to the high suicide rate in Japan, including the economic recession and the rising unemployment rate in Japan (Motohashi, 2012), and psychiatric disorders have been described as one of the most important risk factors for suicide (Cheng, 1995; Hirokawa et al., 2012a). For example, patients with psychosis are at a 12-fold increased risk of completed suicide compared to the general population (Dutta et al., 2010), and it was reported that approx. 5\% of schizophrenic patients commit suicide during their lifetime (Palmer et al., 2005). An unsuccessful suicide attempt was also proposed as a risk factor for completed suicide (Suominen et al., 2004). Certain characteristics of attempted suicide, such as highly lethal methods, might contribute to a higher risk of later completing suicide (Mann et al., 2005).

With respect to the suicide methods used most often in Japan, data from complete suicides with psychiatric disorder suggested that hanging was the most frequent method, followed by jumping from a height (Hirokawa et al., 2012a). In contrast, the data from suicide-attempters with psychiatric disorder admitted to a hospital emergency department in an urban city in Japan revealed that the most common method of suicide was drug overdose, followed by laceration, and then jumping from a height (Nakagawa et al., 2011). Therefore, individuals with a psychiatric disorder who have attempted suicide by jumping from a height or by self-stabbing are frequently seen in emergency departments, and they often suffer from severe physical injuries due to these highly lethal methods.

In order to prevent suicide, it is important to understand the characteristics of suicide attempters, including their methods of suicide. Some earlier investigations found that among individuals who completed a suicide attempt by jumping from a height, there was a higher percentage of schizophrenics and the individuals tended to be younger compared to those committing suicide by other methods 
(Cantor et al., 1989; Reisch et al., 2008). Another research group reported that many survivors of self-stab wounds had a psychotic illness (Gerard et al., 2012). However, with respect to self-stabbing, there are only a few studies, each with a small sample size.

As a result, little is known about differences in diagnostic and demographic characteristics based on methods of suicide among survivors of suicide attempts who have a mental illness, especially between jumping from a height and self-stabbing. The purpose of the present study was to examine the clinical features of patients treated in a psychiatric inpatient unit who attempted suicide by jumping from a height or self-stabbing.

\section{Methods}

\subsection{Subjects}

This was a retrospective study performed at the Osaka General Medical Center (OGMC) located in Osaka, the largest city of western Japan. Over 8.8 million people reside or work in Osaka. There are 768 inpatient beds in the OGMC, including an advanced critical care center and psychiatric inpatient unit. In our psychiatric inpatient unit, patients with combined medical and psychiatric disorders are able to receive psychiatric therapy, physical treatment and rehabilitation simultaneously. The number of similar hospitals in Japan is very limited.

In the present study, the subjects were 202 patients consecutively hospitalized in the psychiatric inpatient unit in OGMC after they attempted suicide between January 1, 2004 and August 31, 2012. We selected those patients who attempted suicide by jumping from a height or self-stabbing. A stab wound was defined as a penetrating wound made by a sharp object, such as a kitchen knife. Patients with only minor self-injuries that did not require hospitalization for inpatient treatment (e.g., a minor wrist cut) were excluded. 
2.2. Assessment of the characteristics of suicide attempters

At least two trained psychiatrists performed face-to-face interviews with all patients and their relative(s) if available, upon admission after the suicide attempt. A psychiatric history was obtained and a physical examination and routine laboratory screening were performed. The psychiatrists made the psychiatric diagnosis, according to the International Classification of Disease, tenth edition (ICD-10) (World Health Organization, 1992). The ICD-10 clinical diagnoses were classified as follows: (a) Schizophrenia spectrum disorders (schizophrenia, schizotypal disorders and delusional disorders, F20-29), (b) Mood disorders (F30-39), (c) Personality disorders (F60-61), (d) Stress-related disorders (including adjustment disorders, F40-48), (e) Organic disorders (F00-09), and (f) Substance-related disorders (mental and behavioral disorders due to psychoactive substance use; F10-19). The patients' ages were classified into under 20,> 20-39, 40-59, and 60 years and older. The following aspects were assessed by interview: 1) the location and method of the suicide attempt, 2) living status (i.e., whether the patient lived alone or with others), 3) family history of suicidal attempt, 4) history of psychiatric hospitalization, 5) history of psychiatric outpatient treatment, 6) previous suicide attempt(s).

\subsection{Statistical analysis}

We used the Mann-Whitney U-test to compare the differences between mean ages and the chi-squared test to compare categorical variables. All tests applied were two-tailed, and $p$-values 0.05 or less were considered significant. We analyzed the data using JMP version 7.0 software (SAS Institute Inc., Cary, NC). 


\subsection{Ethics}

This study was retrospectively conducted by reviewing the medical records of the selected patients.

The Institutional Ethics Committee of Osaka General Medical Center approved this study.

\section{Results}

\subsection{Demographic characteristics}

Table 1 shows the demographic characteristics of the 202 suicide attempters divided into the jumping group and self-stabbing group. The self-stabbing group (mean age 52.3 years) was significantly older than the jumping group (mean age 37.9 years) $(p<0.001)$, and a significantly higher proportion of men were found in the self-stabbing group $(p<0.001)$. Over $70 \%$ of patients in the jumping group had a previous psychiatric history, while less than $50 \%$ of patients in the self-stabbing group had such a history $(p<0.001)$. In contrast, no significant differences between the two groups were found in living status, history of psychiatric hospitalization, history of suicide attempt, or family history of suicide. Among the self-stabbing group, the most common sites of injury were abdomen. The location of jumping suicide attempts was from buildings $(94.0 \%)$ and bridges $(6.0 \%)$. The median height jumped in meters was approx. $13 \mathrm{~m}$ (i.e., 2-3 floor levels) (data not shown).

\subsection{Psychiatric diagnoses}

Table 2 shows the frequencies of psychiatric diagnoses in the jumping and self-stabbing groups. Schizophrenia spectrum disorders were significantly more common in the jumping group $(p<0.05)$, whereas mood disorders were significantly more common in the self-stabbing group $(p<0.001)$. No significant differences between the groups were found in personality disorders, stress-related disorders, organic disorders or substance-related disorders. 


\section{Discussion}

To the best of our knowledge, the present study is the first to compare diagnostic and demographic characteristics between Japanese individuals with mental disorders who attempted suicide by jumping from a height and those who did so by self-stabbing. The suicide attempters who jumped from heights were significantly younger (by 14.4 years) than those who self-stabbed, and they were significantly more likely to be female. Our findings regarding jumping from a height showed the same tendency as that of previous reports (Hunt et al., 2006; Reisch et al., 2008). We also found that among the self-stabbing patients, those aged 60 years and older were the most numerous, and the ratio of males in the self-stabbing group was significantly higher than that in the jumping group. Although in general the frequencies of jumping from a height to commit suicide differ by region and nation, and urban areas tend to have more suicide attempts by jumping from a height, it remains unclear why many jumping-suicide attempters tend to be young and female. Considering the ways of attempting suicide, jumping from a height might be more passive method than self-stabbing. One possible explanation for the young/female jumpers tendency is that they tend to make a choice of passive way without seeing their own blood. In contrast, few studies have reported the clinical features of individuals who attempt suicide by stabbing themselves. The relationships among age, gender and suicide attempts by self-stabbing merit further studies.

We found a significant association between jumping from a height as a suicide method and schizophrenia spectrum disorders. This finding is very similar to those of previous studies (Nowers and Gunnell, 1996; Reisch et al., 2008; Nielssen et al., 2010). However, it has not been satisfactorily explained why patients with schizophrenia frequently chose jumping from a height to attempt suicide. One possible explanation is that hallucinations experienced by schizophrenic individuals may cause 
them to be more likely to commit suicide by jumping off a building or bridge (Cantor et al., 1989). Another study indicated that the severity of psychotic symptoms, such as hallucinations, was not associated with suicide attempts (Bertelsen et al., 2007). Aside from hallucinations, it was reported that death by suicide among schizophrenia patients was associated with a history of depression (Hawton et al., 2005). Similar to our results, Qin (2011) reported that the most commonly diagnosed disorder for suicide victims under 35 years old was schizophrenia and that for suicide victims over 60 years old was recurrent depression.

In the present study, we found a significant association between self-stabbing as a suicide method and mood disorders. Gerard et al. (2012) reported that a significant portion of survivors of self-inflicted stab wounds had a psychotic illness. However, most studies of suicide attempters by self-stabbing were based on small numbers of cases. Our present findings suggest that patients with mood disorders (which may include feelings of guilt) punish themselves by stabbing their own bodies. This method might also be consistent with 'hara-kiri' (ritual suicide by self-disembowelment on a sword) as it is known in Japan.

Hirokawa et al. (2012b) reported that approximately $90 \%$ of suicide completers who received psychiatric treatment had consulted a psychiatrist in the month prior to their suicide. In the present study, most of the patients (132 of the 202 , or $65 \%$ ) had a history of psychiatric treatment prior to admission. In particular, a history of psychiatric treatment was significantly more likely among the suicide attempts by jumping from a height compared to those by self-stabbing. Although we did not have sufficient information about the patients' prior treatment and medication compliance, this finding suggests that the some of the suicide attempts may have been based on a relapse of symptoms due to insufficient or discontinued psychiatric treatment.

This study had several limitations. First, we depended on data from patients admitted to a psychiatric 
inpatient unit in a single hospital in a large urban city, and our results might not apply to suicide attempters who were not admitted for inpatient care or were living in rural areas. Second, this study did not include subjects who completed their suicide attempts. Since jumping from a height and self-stabbing are lethal methods of suicide, some of the potential patient population died before admission. Finally, we did not make a distinction between depressive disorder and bipolar disorder, and with respect to bipolar disorder, it may be necessary to distinguish manic state from depressive state.

Our findings suggest that methods of suicide can differ significantly by psychiatric diagnosis and by gender and age. We propose that suicide prevention plans for psychiatric patients should take these findings into. Further studies are needed to examine the relationship between methods of suicide and mental disorders using larger sample sizes and more detailed clinical information. 


\section{References}

Bertelsen, M., Jeppesen, P., Petersen, L., Thorup, A., Ohlenschlaeger, J., le Quach, P., Christensen, T.O., Krarup, G., Jorgensen, P., Nordentoft, M., 2007. Suicidal behaviour and mortality in first-episode psychosis: the OPUS trial. The British journal of psychiatry. Supplement 51, s140-146.

Cantor, C.H., Hill, M.A., McLachlan, E.K., 1989. Suicide and related behaviour from river bridges. A clinical perspective. The British journal of psychiatry : the journal of mental science 155, 829-835.

Cheng, A.T., 1995. Mental illness and suicide. A case-control study in east Taiwan. Archives of general psychiatry 52, 594-603.

Dutta, R., Murray, R.M., Hotopf, M., Allardyce, J., Jones, P.B., Boydell, J., 2010. Reassessing the long-term risk of suicide after a first episode of psychosis. Archives of general psychiatry 67, 1230-1237.

Gerard, A., de Moore, G., Nielssen, O., Large, M., 2012. Survivors of self-inflicted stab wounds. Australasian psychiatry : bulletin of Royal Australian and New Zealand College of Psychiatrists 20, 44-48.

Hawton, K., Sutton, L., Haw, C., Sinclair, J., Deeks, J.J., 2005. Schizophrenia and suicide: systematic review of risk factors. The British journal of psychiatry : the journal of mental science 187 , 9-20.

Hirokawa, S., Kawakami, N., Matsumoto, T., Inagaki, A., Eguchi, N., Tsuchiya, M., Katsumata, Y., Akazawa, M., Kameyama, A., Tachimori, H., Takeshima, T., 2012a. Mental disorders and suicide in Japan: a nation-wide psychological autopsy case-control study. Journal of affective disorders 140, 168-175.

Hirokawa, S., Matsumoto, T., Katsumata, Y., Kitani, M., Akazawa, M., Takahashi, Y., Kawakami, N., Watanabe, N., Hirayama, M., Kameyama, A., Takeshima, T., 2012b. Psychosocial and psychiatric characteristics of suicide completers with psychiatric treatment before death: a psychological autopsy study of 76 cases. Psychiatry and clinical neurosciences 66, 292-302.

Hunt, I.M., Kapur, N., Robinson, J., Shaw, J., Flynn, S., Bailey, H., Meehan, J., Bickley, H., Burns, J., Appleby, L., Parsons, R., 2006. Suicide within 12 months of mental health service contact in different age and diagnostic groups: National clinical survey. The British journal of psychiatry : the journal of mental science $188,135-142$. 
Mann, J.J., Apter, A., Bertolote, J., Beautrais, A., Currier, D., Haas, A., Hegerl, U., Lonnqvist, J., Malone, K., Marusic, A., Mehlum, L., Patton, G., Phillips, M., Rutz, W., Rihmer, Z., Schmidtke, A., Shaffer, D., Silverman, M., Takahashi, Y., Varnik, A., Wasserman, D., Yip, P., Hendin, H., 2005. Suicide prevention strategies: a systematic review. JAMA : the journal of the American Medical Association 294, 2064-2074.

Motohashi, Y., 2012. Suicide in Japan. Lancet 379, 1282-1283.

Nakagawa, M., Kawanishi, C., Yamada, T., Sugiura, K., Iwamoto, Y., Sato, R., Morita, S., Odawara, T., Hirayasu, Y., 2011. Comparison of characteristics of suicide attempters with schizophrenia spectrum disorders and those with mood disorders in Japan. Psychiatry research 188, 78-82.

Nielssen, O., Glozier, N., Babidge, N., Reutens, S., Andrews, D., Gerard, A., Malhi, G.S., Large, M.M., 2010. Suicide attempts by jumping and psychotic illness. The Australian and New Zealand journal of psychiatry 44, 568-573.

Nowers, M., Gunnell, D., 1996. Suicide from the Clifton Suspension Bridge in England. Journal of epidemiology and community health 50,30-32.

Palmer, B.A., Pankratz, V.S., Bostwick, J.M., 2005. The lifetime risk of suicide in schizophrenia: a reexamination. Archives of general psychiatry 62, 247-253.

Qin, P., 2011. The impact of psychiatric illness on suicide: differences by diagnosis of disorders and by sex and age of subjects. Journal of psychiatric research 45, 1445-1452.

Reisch, T., Schuster, U., Michel, K., 2008. Suicide by jumping from bridges and other heights: social and diagnostic factors. Psychiatry research 161, 97-104.

Suominen, K., Isometsa, E., Suokas, J., Haukka, J., Achte, K., Lonnqvist, J., 2004. Completed suicide after a suicide attempt: a 37-year follow-up study. The American journal of psychiatry 161, $562-563$.

World Health Organization, 1992. International Classification of Mental and Behavioural Disorders: ICD-10. Geneva, Ill: WHO. 
Role of funding source: No funding sources.

Conflict of interest: None of the authors has any conflict of interest to report.

Acknowledgements: We thank Drs. S. Taguchi, A. Haramoto, A. Fukumori, A. Matsumoto, K. Mori, M. Takaya, T. Tomioka and K. Kanai for their psychiatric clinical work. We also thank M. Okada for her contribution to the data collection.

\section{Figures and Tables}

Table 1. Demographic characteristics of suicide attempters by jumping and self-stabbing

Table 2. Psychiatric diagnosis of suicide attempters by jumping and self-stabbing 
Table 1. Demographic characteristics of suicide attempters by jumping and self-stabbing

\begin{tabular}{lcccc}
\multicolumn{1}{c}{ Characteristic } & $\begin{array}{c}\text { Jumping } \\
\mathrm{N}=147(\%)\end{array}$ & $\begin{array}{c}\text { Stabbing } \\
\mathrm{N}=55(\%)\end{array}$ & Test & $\boldsymbol{p}$-value \\
\hline Age, mean (years) & 37.9 & 52.3 & MWU $(\mathrm{Z}=5.16)$ & $<0.001$ \\
Under 20 years & $11(7.5)$ & $4(7.3)$ & Chi & 0.960 \\
20-39 years & $71(48.3)$ & $9(16.4)$ & Chi & $<0.001$ \\
40-59 years & $52(35.4)$ & $20(36.4)$ & Chi & 0.896 \\
60+ years & $13(8.8)$ & $22(40.0)$ & Chi & $<0.001$ \\
Gender (male) & $46(31.3)$ & $32(58.2)$ & Chi & $<0.001$ \\
Living alone & $49(33.3)$ & $17(30.9)$ & Chi & 0.744 \\
Previous psychiatric history & $105(71.4)$ & $27(49.1)$ & Chi & $<0.001$ \\
History of psychiatric hospitalization & $61(41.5)$ & $23(41.8)$ & Chi & 0.967 \\
History of suicide attempt(s) & $43(29.3)$ & $15(27.3)$ & Chi & 0.782 \\
Family history of suicide & $7(4.8)$ & $2(3.6)$ & Chi & 0.730 \\
\hline
\end{tabular}

MWU: Mann-Whitney U-test 
Table 2. Psychiatric characteristics of suicide attempters by jumping and self-stabbing

\begin{tabular}{lcccc}
\multicolumn{1}{c}{ Characteristic } & Jumping & Stabbing & & \\
& $\mathrm{N}=147(\%)$ & $\mathrm{N}=55(\%)$ & Test & $\boldsymbol{p}$-value \\
\hline Schizophrenia spectrum disorders (F20-29) & $52(35.4)$ & $11(20.0)$ & Chi & 0.036 \\
Mood disorders (F30-39) & $30(20.4)$ & $22(40.0)$ & Chi & 0.005 \\
Personality disorders (F60-61) & $26(17.7)$ & $9(16.4)$ & Chi & 0.825 \\
Stress-related disorders (F40-48) & $11(7.5)$ & $6(10.9)$ & Chi & 0.435 \\
Organic disorders (F00-09) & $11(7.5)$ & $3(5.5)$ & Chi & 0.613 \\
Substance-related disorders (F10-19) & $9(6.1)$ & $4(7.3)$ & Chi & 0.767 \\
\hline
\end{tabular}




\section{Acknowledgement:}

We thank Drs. S. Taguchi, A. Haramoto, A. Fukumori, A. Matsumoto, K. Mori, M. Takaya, T.

Tomioka and K. Kanai for their psychiatric clinical work. We also thank M. Okada for her contribution

to the data collection. 


\section{Conflict of Interest:}

None of the authors has any conflict of interest to report. 


\section{Contributors:}

Ryo Kimura designed the study and wrote the protocol.

Shunichiro Ikeda, Hirokazu Kumazaki and Makoto Yanagida managed the literature searches and analyses.

Hidenori Matsunaga undertook the statistical analysis.

Ryo Kimura wrote the first draft of the manuscript.

All authors contributed to and have approved the final manuscript. 


\section{Role of the Funding source:}

There were no external funding sources for this study. 